\title{
Correlation between capillary and venous blood glucose levels in diabetic patients
}

\author{
Muhammad Adnan ${ }^{\mathrm{a}}$, Fakhar Imam ${ }^{\mathrm{b}}$, Iffat Shabbir ${ }^{\mathrm{a}}$, Zahra Ali ${ }^{\mathrm{a}}$, Tayyaba Rahat ${ }^{\mathrm{a}}$ \\ a PMRC Research Centre, Sir Ganga Ram Hospital, Lahore 54000, Pakistan \\ ${ }^{b}$ Fatima Jinnah Medical College, Lahore 54000, Pakistan
}

\begin{abstract}
Background: Rapid blood glucose estimation is required to prescribe treatments and to make dose adjustments in diabetic patients. However, measuring plasma glucose levels is time consuming. Therefore, the use of glucometers has greatly increased.

Objectives: To measure the correlation between capillary and venous blood glucose levels.

Methods: Seventy patients with type 2 diabetes mellitus (T2DM) were enrolled in the present study and informed written consent was obtained from all participants. Demographic characteristics and clinical information was noted. Capillary and venous glucose levels were determined. Statistical Package for Social Sciences version 21 was used for data analysis.

Results: Mean age of patients was $52 \pm 12$ years. It included 29\% men; 71\% women; 9\% smokers; and 90\% poor. Mean venous glucose was $11.73 \pm 4.64 \mathrm{mmol} / \mathrm{L}$ and mean capillary glucose $12.57 \pm 5.21 \mathrm{mmol} / \mathrm{L}$. These findings demonstrated a significant intermethod mean difference of $0.84 \mathrm{mmol} / \mathrm{L}(P<0.001)$. Inter-glucose difference was not significant at glucose levels near normal. However, it increased gradually with rise in glucose measurements and was significant at elevated glucose levels. Both concordance correlation coefficient and intraclass correlation coefficient demonstrated positive correlation and more consistency between glucose estimations. A Bland and Altman plot presented excellent agreement between glucometrically and photometrically determined glucose levels.

Conclusion: A positive correlation coefficient showed strong association between capillary and venous glucose measurements.
\end{abstract}

Keywords: blood glucose, capillary blood glucose, glucometer, plasma blood glucose, self-monitoring of blood glucose

Diabetes mellitus is a major public health problem and its complications can be prevented or delayed by self-monitoring of blood glucose (SMBG). This is a recommended key component of diabetes management by the American Diabetes Association (ADA) [1]. Rapid blood glucose determination is required for prescribing treatments and to make dose adjustments. Laboratory methods to measure plasma glucose levels are time consuming. Therefore, the use of glucometers has greatly increased.

The international organization for standardization (ISO) recommends that total analytical error for a glucometer be within $\pm 15 \mathrm{mg} / \mathrm{dl}$ when laboratory glucose values are $<100 \mathrm{mg} / \mathrm{dl}$; and the acceptable error should be within $15 \%$ for laboratory values $\geq 100$

Correspondence to: Muhammad Adnan, PMRC Research Centre, Sir Ganga Ram Hospital, Lahore 54000, Pakistan.

E-mail: adiprince81@gmail.com $\mathrm{mg} / \mathrm{dl}$. ADA guidelines recommend an analytical error of $\leq 5 \%$ for all values $[2,3]$.

Various studies have been conducted to estimate the accuracy of glucometers in comparison with a standard laboratory method [4-6]. However, limited work has been done in Pakistan regarding the accuracy of glucometers in comparison with laboratory methods in this social setting. Therefore, this study was designed to measure mean differences between glucose measurements determined by the two different methods in Pakistan.

\section{Materials and methods}

This cross-sectional study was approved by the Institutional Ethical Review Board, Fatima Jinnah Medical College/ Sir Ganga Ram Hospital, Lahore on $3^{\text {rd }}$ December 2013 (No. 1156 I.E.R.B.). All patients provided documented informed consent to participate. The study was supported and monitored by the 
Pakistan Medical Research Council. Seventy patients with type 2 diabetes mellitus (T2DM) were enrolled. Demographic characteristics and clinical information was noted. Capillary blood glucose was measured using a Glucometer (On-Call plus, Acon, USA). Venous plasma glucose was measured using a UVVisible spectrophotometer (Microlab 300, Merck, Darmstadt, Germany). Statistical Package for Social Sciences version 21 (IBM SPSS, Armonk, NY, USA) was used for data analysis.

\section{Results}

Mean age of the 70 patients was $52 \pm 12$ years. Participants included 29\% men; 71\% women; 56\% were literate and there were $9 \%$ cigarette smokers. Socioeconomic status of $90 \%$ patients was poor with an average monthly income was 150 USD. Mean duration of diabetes was $12 \pm 08$ years. History of comorbidity showed that $43 \%$ patients had T2DM, $54 \%$ diabetics had hypertension (HTN), and 3\% diabetics had ischemic heart disease (IHD).

Results showed that $99 \%$ of the diabetic participants were monitoring blood glucose. The blood glucose of $70 \%$ patients was being measured by the healthcare providers and in $30 \%$ by family members. Glucometers were used by $64 \%$ patients and glucose levels were measured by laboratory spectrophotometers in 36\% patients. Blood glucose levels were measured at least once in a month in 86\% patients. Only $25 \%$ of patients were monitoring for both fasting and postprandial glucose levels.

The volume used for the Photometric Venous Blood Glucose determination (PVBG) was $11.73 \pm$ $4.64 \mathrm{mmol} / \mathrm{L}$. For the Glucometric Capillary Blood Glucose (GCBG) it was $12.57 \pm 5.21 \mathrm{mmol} / \mathrm{L}$; and for Glucometric Venous Blood Glucose (GVBG) $13.92 \pm 5.69 \mathrm{mmol} / \mathrm{L}$. These findings demonstrate a significant intermethod mean difference of $0.84 \mathrm{mmol} / \mathrm{L}(P<0.001)$. The intermethod glucose difference was not significant at glucose levels near normal. However, the difference increased gradually with a rise in glucose levels and was significant at elevated glucose levels.

Concordance correlation coefficient (CCC) analysis showed strong agreement $(\mathrm{CCC}=0.95)$ between PVBG and GCBG; moderate agreement $(\mathrm{CCC}=0.94)$ was seen between GCBG and GVBG; and poor agreement $(C C C=0.81)$ was present with GVBG and PVBG. Intraclass correlation coefficient (ICC), 0.947 at $95 \% \mathrm{CI}(0.864,0.974)$ showed positive correlation and more consistency between capillary and venous glucose levels (Table 1). For most of the cases a Bland and Altman plot presented excellent agreement between glucometric and spectrophotometric estimations (Figure 1).

Table 1. Comparison of different glucose estimation methods

\begin{tabular}{|c|c|c|c|c|c|c|c|}
\hline & \multicolumn{4}{|c|}{ Paired Differences } & \multirow{3}{*}{$\boldsymbol{P}$} & \multirow{3}{*}{ ICC } & \multirow{3}{*}{ CCC } \\
\hline & \multirow[t]{2}{*}{ Mean } & \multirow[t]{2}{*}{ SD } & \multicolumn{2}{|c|}{$\begin{array}{c}5 \% \text { Confidence Interval } \\
\text { of the Difference }\end{array}$} & & & \\
\hline & & & Lower & Upper & & & \\
\hline PVBG Vs GCBG & 0.84 & 1.40 & 0.51 & 1.17 & $<0.001^{*}$ & $\begin{array}{c}0.947 \\
(0.864-0.974)\end{array}$ & $\begin{array}{c}0.95 \\
(0.505-0.974)\end{array}$ \\
\hline GCBG Vs GVBG & 1.35 & 1.39 & 1.02 & 1.68 & $<0.001^{*}$ & $\begin{array}{c}0.939 \\
(0.643-0.978)\end{array}$ & $\begin{array}{c}0.94 \\
(0.507-0.962)\end{array}$ \\
\hline GVBG Vs PVBG & 2.19 & 1.67 & 1.79 & 2.59 & $<0.001^{*}$ & $\begin{array}{c}0.871 \\
(0.149-0.160)\end{array}$ & $\begin{array}{c}0.87 \\
(0.515-0.887)\end{array}$ \\
\hline
\end{tabular}

${ }^{*} P \leq 0.05$ was considered significant. PVBG, photometric venous blood glucose; GCBG, glucometric capillary blood glucose; GVBG, glucometric venous blood glucose; ICC, intraclass correlation coefficient; CCC, concordance correlation coefficient 

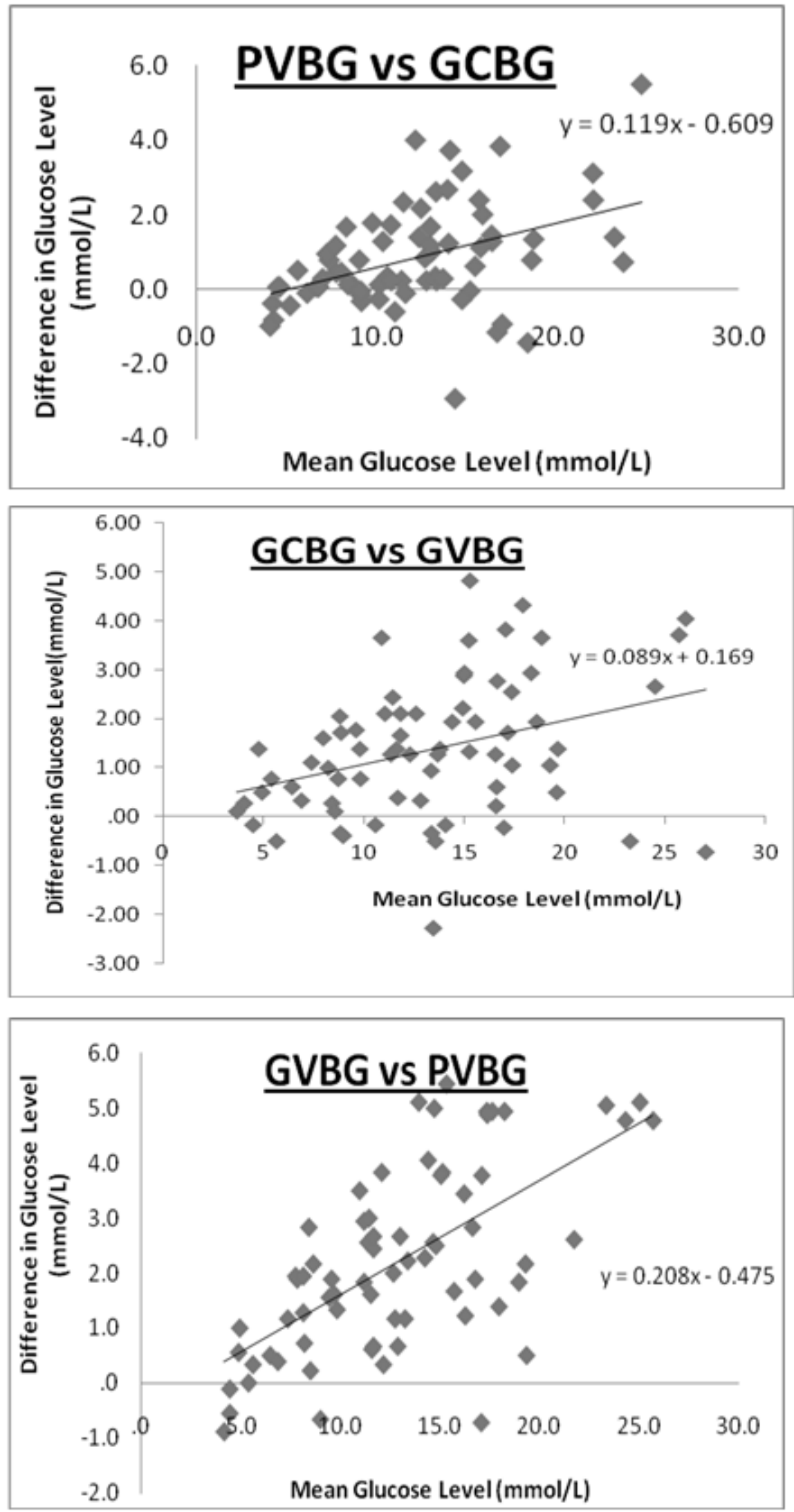

Figure 1 Bland and Altman plots

PVBG, photometric venous blood glucose; GCBG, glucometric capillary blood glucose; GVBG, glucometric venous blood glucose 


\section{Discussion}

Monitoring of blood glucose on regular basis is very useful in preventing hypoglycemia and adjusting medications. In the present study, 98.6\% patients underwent regular glucose measurements [1]. It is well established that the education and training of glucometer operators is important to obtain accurate results. However, in our study, $44 \%$ of patients were illiterate. The training status of all glucometer operators included healthcare providers and family members, but details remained unknown. Raine et al. concluded that up to $16 \%$ of patients do not use glucometers properly and this leads to $\pm 33 \%$ errors [7].

Comparison of photometric venous and glucometric capillary glucose estimations resulted in a mean difference of $0.84 \mathrm{mmol} / \mathrm{L}$, which was greater than the $0.58 \mathrm{mmol} / \mathrm{L}$ reported by Boyd et al. [8], but it was comparatively lower than the two other mean differences calculated in the study of Bina et al., who studied differences in blood glucose levels at various sample sites (forearm, palm, and thigh) compared with fingertip capillary blood. They found significant differences $(P<0.05)$ [9]. Similar significant differences $(P<0.001)$ in blood glucose measurement at forearm with respect to fingertip capillary blood glucose was obtained in their study. Other than various sample sites, factors that may contribute to this significant difference include hematocrit and $\mathrm{pH}$ of blood, drugs, triglycerides, and bilirubin [10].

When venous blood was tested using a glucometer, relatively poor agreement $(\mathrm{CCC}=0.81)$ was observed. The same was reported by Funk et al. [6], because glucometers are actually designed only for capillary blood. Yang et al. have observed insignificant inter-measurement glucose differences at glucose levels near normal and significant differences at elevated levels [11]. Similar findings were reported in the present study, which verified the results by Yang et al. Significant differences at elevated levels may result from reagents and/or instruments that may produce invalid results beyond limits set by the manufacturer.

The correlation coefficients i.e. CCC $=0.95$ and ICC $=0.947$ for capillary and venous glucose estimations obtained in this study were very close and comparable to correlation coefficients for different glucometers reported by Jamaluddin et al. [12]. These findings support that capillary blood glucose by glucometers can be determined at point of care, but certain conditions like glucometer operator's training and participation in quality assurance programs should be considered.

\section{Conclusion}

A positive correlation coefficient showed strong association between capillary and venous glucose measurements including large percentage of illiterates.

\section{Acknowledgments}

We thank Mr. Asif Javed, Mr. Ghulam Nabi, and Mr. Muhammad Imran Talib who provided technical help in glucose testing.

The authors have no conflict of interest to report.

\section{References}

1. American Diabetes Association. Standards of medical care in diabetes. Diabetes Care. 2012; 35:S11-63

2. Freckmann G, Schmid C, Baumstark A, Pleus S, Link M, Haug C. System accuracy evaluation of 43 blood glucose monitoring systems for self-monitoring of blood glucose according to DIN EN ISO 15197. J Diabetes Sci Technol. 2012; 6:1060-75.

3. Aghakachoei S, Zargarzadeh AH, Amini M. Comparison of blood glucose values using two glucose meters and standard laboratory method in hospitalized patients in a teaching hospital. J Pharm Care. 2014; 2:15-21.

4. Schrot RJ, Patel KT, Foulis P. Potential variables altering home glucose measurement. Evaluation of inaccuracies in the measurement of glycemia in the laboratory, by glucose meters, and through measurement of hemoglobin A1c. Clin Diabetes. 2007; 25:243-9.

5. Hortensius J, Slingerland RJ, Kleefstra N, Logtenberg SJJ, Klaas, Groenier KH, et al. Self monitoring of blood glucose: the use of the first or the second drop of blood. Diabetes Care. 2011; 34:556-60.

6. Funk D, Chan L, Lutz N, et al. Comparison of capillary and venous glucose measurements in healthy volunteers. Prehosp Emerg Care. 2001; 5:275-7.

7. Raine CH, Schrock LE, Edelman SV, Mudaliar SRD, Zhong W, Proud LJ, et al. Significant insulin dose errors may occur if blood glucose results are obtained from miscoded meters. J Diabetes Sci Technol. 2007; 1:205-10.

8. $\quad$ R Boyd, B Leigh, P Stuart. Capillary versus venous bedside blood glucose estimations. Emerg Med J. 2005; 22:177-9.

9. Bina DM, Anderson RL, Johnson ML, Bergenstal RM, Kendall DM. Clinical impact of prandial state, exercise, 
and site preparation on the equivalence of alternativesite blood glucose testing. Diabetes Care. 2003; 26: 981-5.

10. Narendra Kotwal, Aditi Pandit. Variability of capillary blood glucose monitoring measured on home glucose monitoring devices. Indian J Endocrinol Metab. 2012; 16:S248-51.

11. Yang C, Chang C, Lin J. A comparison between venous and finger-prick blood sampling on values of blood glucose. IPCBEE. 2012; 39:206-10.

12. Jamaluddin FA, Gunavathy M, Yean CY, Thevarajah M. Variability of point-of-care testing blood glucometers versus the laboratory reference method in a tertiary teaching hospital. Asian Biomed. 2012; 6 : 67-74. 